\title{
CBD-enriched cannabis for autism spectrum disorder: an experience of a single center in Turkey and reviews of the literature
}

\author{
Serap Bilge ${ }^{1^{*}}$ (D) and Barış Ekici ${ }^{2}$
}

\begin{abstract}
Introduction: Autism spectrum disorder is a neurodevelopmental disorder characterized by deficits in communication, social interaction, restricted interest, and repetitive behaviors. Although more cases are being diagnosed, no drugs are approved to treat the core symptoms or cognitive and behavioral problems associated with autism. Therefore, there is an urgent need to develop an effective and safe treatment.
\end{abstract}

Objective: In this study, we aim to share our 2-year experience with CBD-enriched cannabis treatment in autism and review the latest studies.

Materials and methods: The study included 33 (27 males, six females) children diagnosed with autism spectrum disorder who were followed up between January 2018 and August 2020. The mean age was $7.7 \pm 5.5$ years. The average daily dosage of cannabidiol (CBD) was $0.7 \mathrm{mg} / \mathrm{kg} /$ day $(0.3-2 \mathrm{mg} / \mathrm{kg} /$ day). The median duration of treatment was 6.5 months (3-28 months). The preparations used in this study contained full-spectrum CBD and trace elements tetrahydrocannabinol (THC) of less than $3 \%$.

Results: The outcomes were evaluated before and after treatment based on clinical interviews. At each follow-up visit, parents were asked to evaluate the effectiveness of the CBD-enriched cannabis treatment. According to the parents'reports, no change in daily life activity was reported in 6 (19.35\%) patients. The main improvements of the treatment were as follows: a decrease in behavioral problems was reported in 10 patients (32.2\%), an increase in expressive language was reported in 7 patients (22.5\%), improved cognition was reported in 4 patients (12,9\%), an increase in social interaction was reported in 3 patients (9.6\%), and a decrease in stereotypes was reported in 1 patient (3.2\%). The parents reported improvement in cognition among patients who adhered to CBD-enriched cannabis treatment for over two years. The antipsychotic drug could be stopped only in one patient who showed mild ASD symptoms. No change could be made in other drug use and doses. Additionally, this study includes an extensive review of the literature regarding CBD treatment in autism spectrum disorder. According to recent studies, the average dose of CBD was $3.8 \pm 2.6 \mathrm{mg} / \mathrm{kg} /$ day. The ratio of CBD to THC in the used preparations was 20:1. The most significant improvements were seen in the behavioral problems reported in $20-70 \%$ of the patients.

Conclusion: Using lower doses of CBD and trace THC seems to be promising in managing behavioral problems associated with autism. In addition, this treatment could be effective in managing the core symptoms and cognitive

*Correspondence: sarabsmustafa@hotmail.com

1 Department of Pediatric Neurology, Çukurova Medical School, 01790 Balcalı, Turkey

Full list of author information is available at the end of the article original author(s) and the source, provide a link to the Creative Commons licence, and indicate if changes were made. The images or other third party material in this article are included in the article's Creative Commons licence, unless indicated otherwise in a credit line to the material. If material is not included in the article's Creative Commons licence and your intended use is not permitted by statutory regulation or exceeds the permitted use, you will need to obtain permission directly from the copyright holder. To view a copy of this licence, visit http://creativecommons.org/licenses/by/4.0/. 
functions. No significant side effects were seen at the low doses of CBD-enriched cannabis when compared to other studies.

Keywords: Autism spectrum disorder, Cannabidiol, Cannabis

\section{Background}

Autism spectrum disorder (ASD) is a neurodevelopmental disorder that varies in severity and is characterized by deficits in communication, social interaction, restricted interest, and repetitive behaviors (Fusar-Poli et al. 2020). During the last three decades, there has been a threefold increase in the number of children diagnosed with ASD (Lihi Bar-Lev Schleider et al. 2019). Currently, it affects up to 1 in 54 individuals (Maenner et al. 2020). Cooccurring medical conditions such as epilepsy, intellectual disability, and behavior problems occur in these individuals (Pretzsch et al. 2019a; Pretzsch et al. 2019b).

The etiopathogenesis of ASD remains largely unknown. Several genetic, perinatal, and environmental factors seem to be involved. Some researchers have evidenced an imbalance in the endogenous neurotransmission system, such as the serotoninergic, $\gamma$ aminobutyric acid (GABA), and endocannabinoid system (ECS), which regulate functions such as emotional responses and social interactions typically impaired in ASD (Fusar-Poli et al. 2020).

Endocannabinoids (eCBs) and their receptors are present in the nervous system, connective tissue of internal organs, glands, and immune system. Cannabinoid receptor 1 (CB1) is a G protein-coupled receptor (GPR) that is found mainly in the central nervous system (Mc Partlan et al. 2014). In mammals, high concentrations of CB1 are found in the brain area that regulates appetite, memory, fear extinction, motor responses, and postures such as the hippocampus, basal ganglia, basolateral amygdala, hypothalamus, and cerebellum (Aran et al. 2019; Mc Partlan et al. 2014). CB1 can also be found in nonneuronal cells. Data indicate that cannabinoid receptor type 2 (CB2) is linked to a variety of immune functional events. However, it may play a functionally relevant role in the central nervous system (Aran et al. 2019; Bridgemanan and Abazia 2017).

There are two endogenous cannabinoids, $\mathrm{N}$-arachidonoylethanolamine (anandamide) and two arachidonoylglycerols (2-AG). The ECS has been broadened by discovering new secondary receptors, ligands, and ligand metabolic enzymes, including transient receptor potential cation channel subfamily V member 1 (TRPV1) (Mc Partlan et al. 2014).

Anandamide and 2-AG can act via CB1 and CB2 receptors and exert a range of biological effects in central and peripheral cells. Anandamide is broken down by fatty acid amide hydrolase (FAAH); inhibitors of FAAH lead to an increase in anandamide. CBD act as an inhibitor of FAAH (Bridgemanan and Abazia 2017). Endocannabinoid signaling occurs in a retrograde direction; that is, signaling is initiated in postsynaptic neurons and acts upon presynaptic terminals. In contrast to classical neurotransmitters, eCBs are not stored. They are produced on demand upon stimulation of postsynaptic cells (Aran et al. 2019; Zamberletti et al. 2017).

Interestingly, CBD displays a low affinity for CB1 and $\mathrm{CB} 2$ receptors. $\mathrm{CBD}$ facilitates excitatory glutamate and inhibitory GABA neurotransmission across the brain through agonism at the TRPV1 receptor (Pretzsch et al. 2019a; Mc Partlan et al. 2014). Additionally, CBD can increase GABAergic transmission by antagonizing $\mathrm{G}$ protein-coupled receptor 55 (GPR55), especially in the basal ganglia. CBD is thought to be an agonist at prefrontal serotonin $5-\mathrm{HT}_{1 \mathrm{~A}}$ receptors (Castillo et al. 2012) (Fig. 1).

Another mechanism of action can be via vasopressin and oxytocin. The presence of oxytocin in the CSF seems to originate from neuronal oxytocinergic extensions to the limbic system, brain stem, and spinal cord. Oxytocin receptors are distributed in different parts of the central nervous system, such as the basal ganglia, limbic system, thalamus and hypothalamus, and brain stem. Oxytocin modulates social behavior, motor function, pain control, memory and learning, eating behavior, stress and anxiety, and emotional processing. Oxytocin administration reduces stress and anxiety and depression in animal models. This effect seems to be modulated at least partly by the effects of oxytocin on the hypothalamic-pituitaryadrenal (HPA) axis and the opioidergic and dopaminergic systems in limbic brain structures. Several animal model studies support the role of oxytocin in improving social behavior, an effect that appears to involve the melatoninergic and endocannabinoid systems, specifically an increase in social interactions produced by agonism at the melanocortin four receptor (MC4R (Russo et al. 2005; Dos Santos et al. 2019). CBD leads to enhancement in the release of vasopressin and oxytocin; thus, it could positively affect ASD core symptoms. Studies have shown that oxytocin administration to patients with ASD improves social interactions, reduces classic repetitive behavior, and increases eye contact (Weia et al. 2015). Another mechanism of action of CBD is to act as a dopamine receptor antagonist, which can facilitate its use as an antipsychotic (Dos Santos et al. 2019; Weia et al. 2015). 


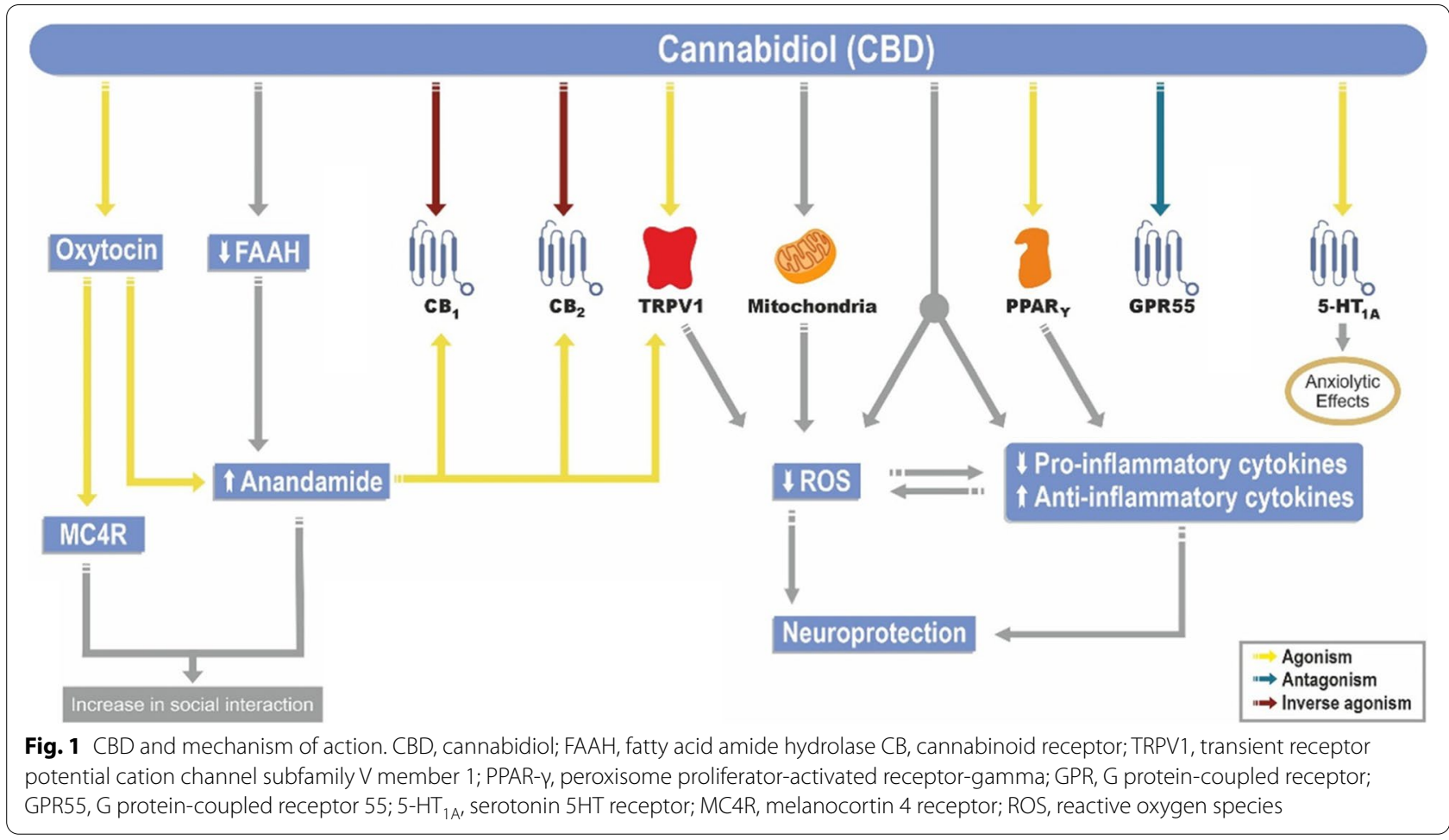

CBD may act as a neuroprotectant against mitochondrially acting toxins (Davies and Bhattacharyya 2019; Bartova and Birmingham 1976). The highly lipophilic aspect of CBD gives them access to intracellular sites of action. Many studies have suggested mitochondria as targets for CBD, and many theories are based on this idea; one of these theories is that the outer mitochondrial membrane has $\mathrm{CB} 1$ receptors. This theory reveals that CBD affects the function of the cells by establishing homeostasis and influencing mitochondria and energy production (Bartova and Birmingham 1976; Ryan et al. 2009).

THC is known to be a major psychoactive component of Cannabis. THC is a partial agonist at CB1 and CB2 (Ryan et al. 2009). Signals through transducing G-proteins and activation of these G-proteins by THC cause inhibition of adenyl cyclase activity, the closing of voltage-gated calcium channels, and the opening of inward rectifying potassium channels. The psychoactive nature of THC limits its use due to side effects. However, a varied mixture of THC with other phytocannabinoids with very weak or no psychoactivity quality has started to be used as a therapeutic drug in humans (Bloomfield et al. 1982; Rodríguez De Fonseca et al. 1992). In this study, we aim to share our 2-year experiences with CBD-enriched cannabis treatment in autism and review the latest studies.

\section{Methods and materials \\ Patients}

This research was conducted in accordance with the Declaration of Helsinki at the Pediatric Clinics of Neurology in Istanbul. CBD-enriched cannabis treatment was started in 54 patients who were diagnosed with ASD. The study included 33 (27 males, six females) children diagnosed with autism spectrum disorder who were followed up between January 2018 and August 2020. The diagnosis of ASD was based on DSM V criteria (American Psychiatric Association 2013). Twentyone participants refused to participate in this study. The most common reasons for not participating in the study were fear of adverse effects, cost of CBD-enriched cannabis, bitter taste, and behavioral problems. The mean age of the non-participating 21 children was $7.2 \pm 4.2$. Ten patients had mild, while 11 had severe autism according to the DSM V. Four patients were female, and 17 were male. Three children had abnormal EEG, and one was diagnosed with epilepsy, and he was on valproic acid treatment. Three patients attended mainstream schools and received their education there, while eighteen patients had intellectual disabilities. All non-participating 21 ASD patients used antipsychotic drugs. Sixteen patients used risperidone, and five patients used aripiprazole. The median duration of antipsychotic drug administration was $8.2 \pm 2.6$ 
months. The median duration of follow-up was 4.4 $1 \pm$ 1 years.

Informed consent was obtained from the parents of all children participating in the study. The mean age of the participating 33 children was $7.7 \pm 5.5$. Fifteen patients had mild autism, while 18 had severe autism according to the DSM V. Three patients were diagnosed with epilepsy before starting CBD-enriched cannabis; two of them used oxcarbazepine, while one used valproic acid. Seven patients had abnormal electroencephalography (EEG) results without any episodes of previous seizures. Five patients attended mainstream schools and received their education there, while twenty-eight patients had intellectual disabilities and attended schools that catered to special educational needs. Two patients were using CBD-enriched cannabis for over two years. There was no predefined duration of this treatment in our patients. All ASD patients used antipsychotic drugs. Twenty-six patients used risperidone, and seven patients used aripiprazole. The median duration of antipsychotic drug administration was $8.5 \pm 2.3$ months. All the patients were provided with psychosocial treatment. The median duration of follow-up was $4.6 \pm 1.3$ years. There were no significant differences between the 2 group profiles (participating and non-participating) regarding sex ratio, median age, and autism severity.

\section{Treatment}

The legal basis for using cannabis-related drugs is not fully apparent in Turkey, and a maximum of $0.3 \%$ THC is allowed to be used in these preparations. Due to the lack of availability and difficulty of access to these therapeutic preparations, various cannabis strains of CBD-enriched cannabis extracts have been used. The two CBD-enriched cannabis brands used were CBDistillery and CBDodgamax. Both had similar available forms of drops of 500 , 1000 , and $2500 \mathrm{mg} / 30 \mathrm{ml}$ and contained full-spectrum CBD and trace THC. These drops were started with dosages that were calculated according to the patient's body weight, with one sublingual drop twice a day and one drop every three days. The average daily CBD-enriched cannabis dose was $0.7 \mathrm{mg} / \mathrm{kg}(0.3-2 \mathrm{mg} / \mathrm{kg})$. No patient was given a daily maintenance dose of CBD higher than $40 \mathrm{mg} /$ day. The average duration of treatment was 6.5 months (3-28 months).

\section{Results and outcomes}

The outcomes were evaluated before and after treatment based on clinical interviews. At each follow-up visit, parents were asked to assess the overall effectiveness of CBD-enriched cannabis treatment. According to the parents' reports, no change in daily life activity was reported in $6(19.35 \%)$ patients. The main improvements of the treatment were as follows: a decrease in behavioral problems was reported in 10 patients (32.2\%), an increase in expressive language was reported in 7 patients $(22.5 \%)$, improved cognition was reported in 4 patients (12.9\%), an increase in social interaction was reported in 3 patients (9.6\%), and a decrease in stereotypes was reported in 1 patient (3.2\%). The parents reported improvement in cognition in patients who adhered to CBD-enriched cannabis treatment for over two years. The antipsychotic drug could be stopped only in one patient who showed mild ASD symptoms. No change could be made in other drug use and doses.

\section{Discontinuation and side effects}

A 13-year-old male patient with severe autism had generalized seizures after using $5 \mathrm{mg}$ sublingual CBD, and the drug was discontinued because of this side effect. The epileptic seizures persisted despite the discontinuation of the treatment. Interictal sleep EEG showed symmetrical bilateral frontotemporal sharp-slow wave complexes. The patient was regularly treated with valproic acid and remained seizure-free after starting this antiepileptic drug. CBD-enriched cannabis was also discontinued in a nine-year-old male patient with severe autism after two weeks because of a significant increase in stereotypes. No change in laboratory values related to CBD-enriched cannabis was found in any patient.

Restlessness was the only reported side effect in 7 (22\%) out of 31 patients who continued treatment for at least three months, and the CBD-enriched cannabis dose was reduced in these patients. As the amount was reduced, restlessness decreased.

\section{A review of other studies}

The popularity of CBD-enriched cannabis for the treatment of autism is increasing. Scoping reviews were done to achieve a broad and thorough examination of the literature in this area. Aran et al. (2019) were the first to retrospectively assess CBD-enriched cannabis effects on 60 children with ASD and severe behavioral problems using an open-label cohort study. The mean age was $11.8 \pm$ 3.5 years; $82 \%$ of patients used psychiatric medications; $77 \%$ of patients had low cognitive function; and $23.3 \%$ of patients had epilepsy. All the children received CBD and THC in a 20:1 ratio. The mean total daily dose was $3.8 \pm$ $2.6 \mathrm{mg} / \mathrm{kg} /$ day CBD and $0.29 \pm 0.22 \mathrm{mg} / \mathrm{kg} /$ day THC for children who received three daily doses $(n=44)$ and 1.8 $\pm 1.6 \mathrm{mg} / \mathrm{kg} / \mathrm{day}$ CBD and $0.22 \pm 0.14 \mathrm{mg} / \mathrm{kg} /$ day THC for children who received two daily doses $(n=16)$. The doses were titrated over 2-4 weeks. The mean followup period was $10.9 \pm 2.3$ months. Efficacy was assessed using the Caregiver Global Impression of Change (CaGI) scale. Considerable improvement in behavioral problems 
was noticed in $61 \%$ of patients. Improvement in anxiety and communication problems was seen in 39 and 47\%, respectively. Based on these promising results, Aren et al. launched a new placebo-controlled crossover trial. This study is ongoing, and new outcomes will be addressed in future publications (Aran et al. 2019).

Another study was conducted to evaluate the efficacy and safety of CBD-enriched cannabis effects on autism. This prospective, open-label study was carried out by Lihi Bar-Lev Schleider et al. and included 188 patients. The mean age was $12.9 \pm 7$ years. A total of $14.4 \%$ of patients had epilepsy. Most patients used preparations with $30 \%$ CBD and 1.5\% THC, and the average concentrations of $\mathrm{CBD}$ and THC were $79.5 \pm 61.5 \mathrm{mg}$ and $4.0 \pm 3.0 \mathrm{mg}$, respectively. After one month of treatment, 179 patients adhered to the treatment, and only 119 patients could be evaluated. Significant improvement was reported in $48.7 \%$ of patients, moderate improvement was reported in $31.1 \%$ of patients, and no change was reported in $14.3 \%$ of patients. Side effects were reported in $5.9 \%$ of patients. After 6 months of treatment, 155 patients continued treatment with CBD. Of the latter group, 93 patients responded to the questionnaire, $30.1 \%$ reported significant improvement, $53.7 \%$ reported moderate improvement, $6.4 \%$ reported slight amelioration, and $8.6 \%$ of the patients reported no change. Quality of life, mood, and ability to perform daily living activities were evaluated before the treatment and at 6 months. A total of 31.3\% of the patients reported good quality of life before treatment. After 6 months, this percentage increased up to 66.8\% (Lihi Bar-Lev Schleider et al. 2019).

Paulo Fleury et al. (2019) conducted a prospective, observational, and open-label study with a cohort of 18 autistic patients who received CBD-enriched cannabis (with a CBD-to-THC ratio of 75/1). The average dose of CBD was $4.55 \mathrm{mg} / \mathrm{kg} /$ day (a minimum of $3.75 \mathrm{mg}$ and a maximum of $6.45 \mathrm{mg} / \mathrm{kg} /$ day). The average THC dose was $0.06 \mathrm{mg} / \mathrm{kg} /$ day (a minimum of 0.05 and a maximum of $0.09 \mathrm{mg} / \mathrm{kg} /$ day). The mean age was ten years. Fifteen patients adhered to the treatment (10 nonepileptic and five epileptic), and only one patient showed a lack of improvement in autistic behaviors. The most significant improvements were reported for seizures, attention-deficit/hyperactivity disorder, sleep disorders, communication, and social interaction (Paulo Fleury et al. 2019). Barchel et al. (2019) performed an open-label study on 53 autistic children. The median age was 11 (4-22) years; these patients received CBD at a concentration of $30 \%$ and a 1:20 ratio of CBD to THC. The median THC interquartile range (IQR) daily dose was 7 (4-11) $\mathrm{mg}$, and the median CBD (IQR) daily dose was 90 (45-143) $\mathrm{mg}$. The median duration of treatment was 66 days (30-588). Self-injury and rage attacks improved by $67.6 \%$ and worsened by $8.8 \%$, respectively. Improvement in hyperactivity symptoms was reported in $68.4 \%$ of patients, $28.9 \%$ reported no change, and $2.6 \%$ reported worsening symptoms. Sleep problems improved by $71.4 \%$ and worsened by $4.7 \%$. There was an improvement in anxiety in $47.1 \%$ and worsening in 23.5\% of patients (Barchel et al. 2019). Mojdeh Mostafavi et al. (2020) reported positive effects of cannabis in ASD, especially in aggressive and self-injurious behaviors (Mostafavi and Gaitanis 2020). McVige et al. (2020) carried out an important retrospective and open-label study on 20 patients with ASD (6 with epilepsy and 14 with pain). These patients were on cannabis treatment. The study reported very significant positive outcomes. The Autism/Caregiver Global Impression of Change (ACGIC) scale revealed improvements in sleep, mood, and aggression toward the self or others; there were also improvements in patient communication abilities and attention/concentration (McVige et al. 2020).

According to Aren et al.s study, adverse events such as hypervigilance aggravated sleep disturbances in $14 \%$ of patients. This side effect was resolved by omitting or adjusting the evening doses. Irritability in $9 \%$ and loss of appetite in $9 \%$ were seen. A thirteen-year-old girl received $6.5 \mathrm{mg} / \mathrm{kg} /$ day $\mathrm{CBD}$ and no other medications; when she gradually increased the THC dose up to $0.72 \mathrm{mg} / \mathrm{kg} / \mathrm{day}$, she developed sudden behavioral changes such as unusual vocalization and refusal to sleep and eat for two days. The symptoms resolved when she stopped CBD and THC and received antipsychotic treatment (ziprasidone). After cannabis treatment, psychiatric medications were regulated in most patients; $33 \%$ received fewer or lower doses, $24 \%$ stopped taking medications, and $8 \%$ received more medication or higher doses (Aran et al. 2019). Lihi BarLev Schleider et al. reported mild side effects such as restlessness, sleepiness, dry mouth, and digestion problems (Lihi Bar-Lev Schleider et al. 2019). Paulo Fleury et al. reported that three patients stopped using CBDenriched cannabis in a period shorter than one month due to side effects (autistic behaviors had worsened in two patients, which might happen due to the unsupervised and sudden cessation of the antipsychotics; one patient had insomnia, irritability, increased heart rate, and worsening of psych-behavioral crises that might be due to the interaction of cannabis with previous prescribed antipsychotic drugs). Mild and transient adverse effects such as sleepiness, moderate irritability, diarrhea, increased appetite, conjunctival hyperemia, and increased body temperature were also reported (Paulo Fleury et al. 2019). 


\section{Discussion}

In the updated review, preliminary evidence announcing that cannabinoids (compounds with different ratios of CBD and THC) could exert beneficial effects on some ASD-associated symptoms, such as behavioral problems, hyperactivity, and sleep disorders, with a lower number of metabolic and neurological side effects than approved medications. Importantly, treatment with cannabinoids permits a reduction in the number of prescribed drugs and significantly reduces the frequency of seizures in participants with comorbid epilepsy. In this paper, we aimed to make some critical points related to the main findings and mechanisms of action of cannabinoids, such as a decrease in behavioral problems, an increase in the expressive language, an improvement in cognition, and an increase in social interaction when patients used CBDenriched cannabis at a dose of $0.7 \mathrm{mg} / \mathrm{kg}(0.3-2 \mathrm{mg} / \mathrm{kg})$, which is lower than the doses reported in other studies. Furthermore, these results are consistent with other studies that suggest that supplementing ASD patients with CBD-enriched cannabis could improve behavioral problems. A dose of $3.8 \pm 2.6 \mathrm{mg} / \mathrm{kg} /$ day CBD was used in Aren et al's study and yielded improvements in anxiety and communication problems. According to Paulo Fleury et al., the average dose of CBD was $4,55 \mathrm{mg} / \mathrm{kg} /$ day, and the results showed that only one patient reported no improvement in autistic behaviors. The most significant improvements were reported for seizures, attention-deficit/hyperactivity disorder, sleep disorders, communication, and social interaction. In addition, improvements in expressive language were seen. $\mathrm{CBD}$-enriched cannabis might help children with ASD via several possible mechanisms, including its anxiolytic and antipsychotic properties and its impact on the endocannabinoid system (ECS) and oxytocin (Dos Santos et al. 2019; McVige et al. 2020; Premolia et al. 2019). According to our results, we recommend using lower doses of CBD-enriched cannabis.

CBD use is not devoid of health risks; known risks include liver damage, adverse effects on the male reproductive system, potential drug interactions that may be associated with adverse events or diminished efficacy of approved therapies, and additional unknown health risks. However, the pharmacology of CBD has not been well studied; thus, little is known about both the potential therapeutic benefits and the hazards of short- or long-term use (Leas et al. 2020). According to our study, restlessness was the only mild side effect seen in some patients which was resolved on making some doses adjustments. In addition, generalized seizures after starting CBD-enriched cannabis. And these seizures reoccurred even several months after cessation of CBD treatment, and abnormal EEG results were seen. Therefore, this study cannot make causal inferences on the relation between CBD-enriched cannabis and seizures. Not all patients benefit equally from the use of CBD. The reason why some patients experienced benefits while others experienced side effects could be due to candidate genes that may influence the acute effects of cannabis. Genes posited to have specific influences on cannabis include CNR1, CB2, FAAH, MGL, TRPV1, and GRP55. When some patients have a mutation in these receptors, different results could be seen when cannabis was used (Agrawal and Lynskey 2009). Other studies also reported reversible and some mild side effects, none of which were life-threatening. Most of the side effects were overcome by adjusting the doses. Furthermore, the use of recreational cannabis in adolescents is associated with several risks, including decreased motivation, addiction, mild cognitive decline, and schizophrenia. However, these complications are all attributed to THC. Our study drug was full-spectrum CBD and trace THC. Nevertheless, systematic evaluation of safety data of CBD use in children is still lacking. Future research is recommended that examines the clinical impact of CBD-enriched cannabis. Additionally, rarer side effects were seen in our patients compared to other studies, which could be due to using lower doses of CBD and trace THC (a brief overview of all these studies is given in Tables 1 and 2).

These preclinical data and the current study results render further exploration of this treatment avenue in controlled studies. Until such evidence is available, physicians should be cautious when using medical cannabis to treat children with ASD since initial reports of promising treatment in children with ASD are often found.

\section{Limitations of the study}

The absence of the control study group, the use of various strains of CBD-enriched cannabis extracts, different durations of treatment and dosages, and depending on the reports of the parents instead of standard assessment scales are considered to be the main limitations of the study. The clinical assessments were done with knowledge of the patients' treatment (it was an open-label case series, not a blinded clinical trial.

\section{Conclusion}

Using lower doses of CBD and trace THC seems to be promising in the management of behavioral problems associated with autism. In addition, this treatment could be effective in managing core symptoms and cognitive functions. No significant side effects were seen at the low doses of CBD-enriched cannabis when compared to other studies. 


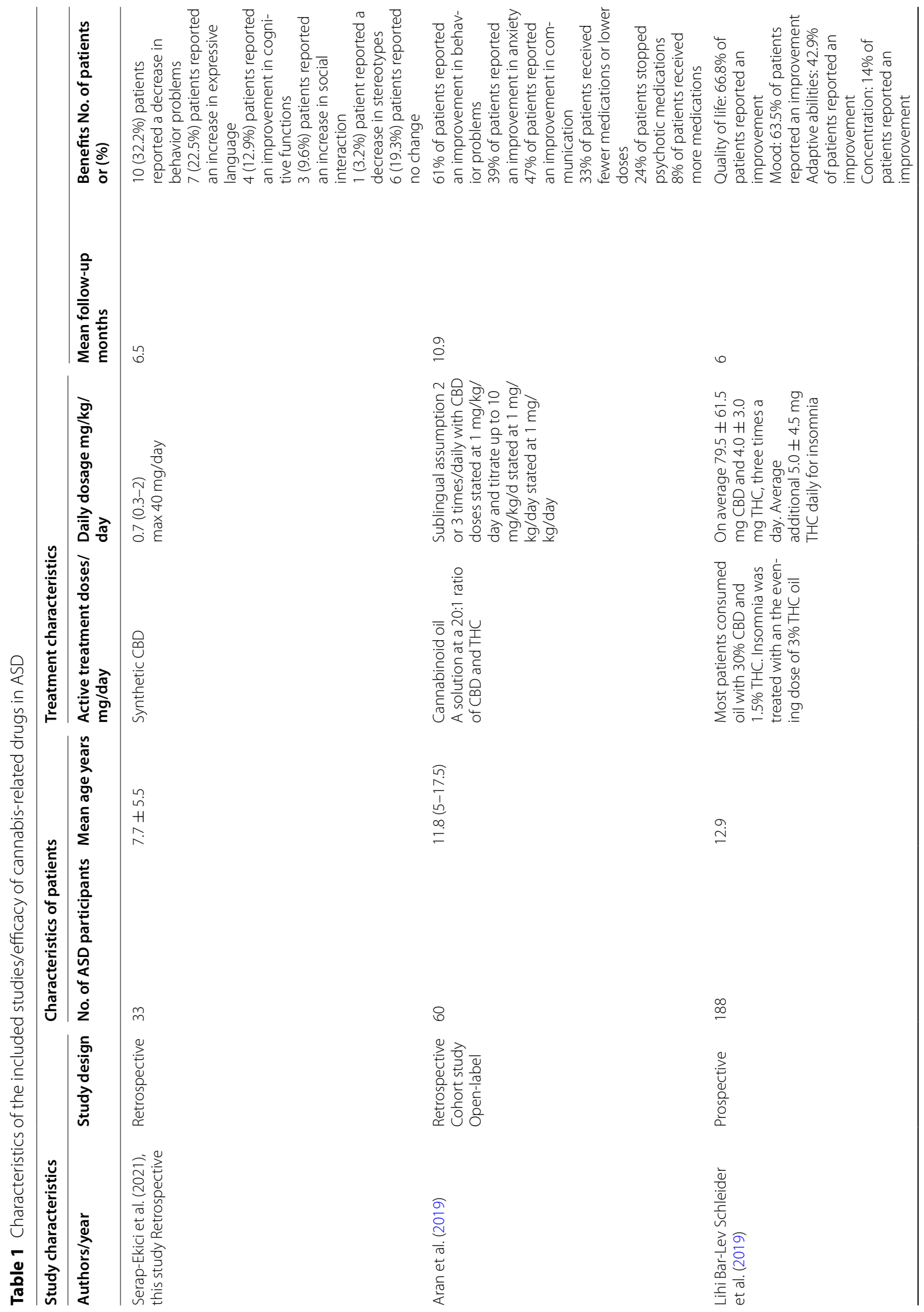




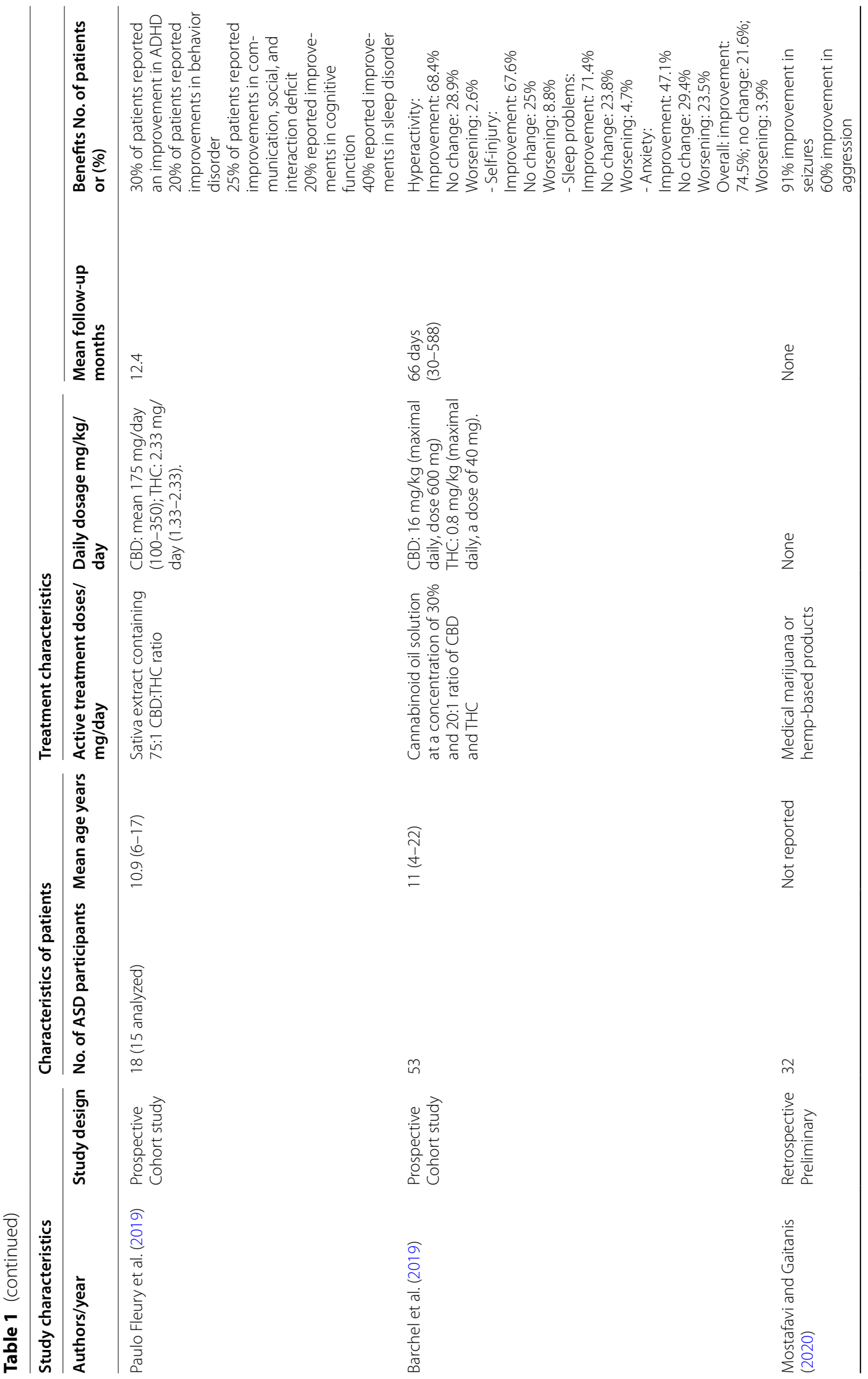




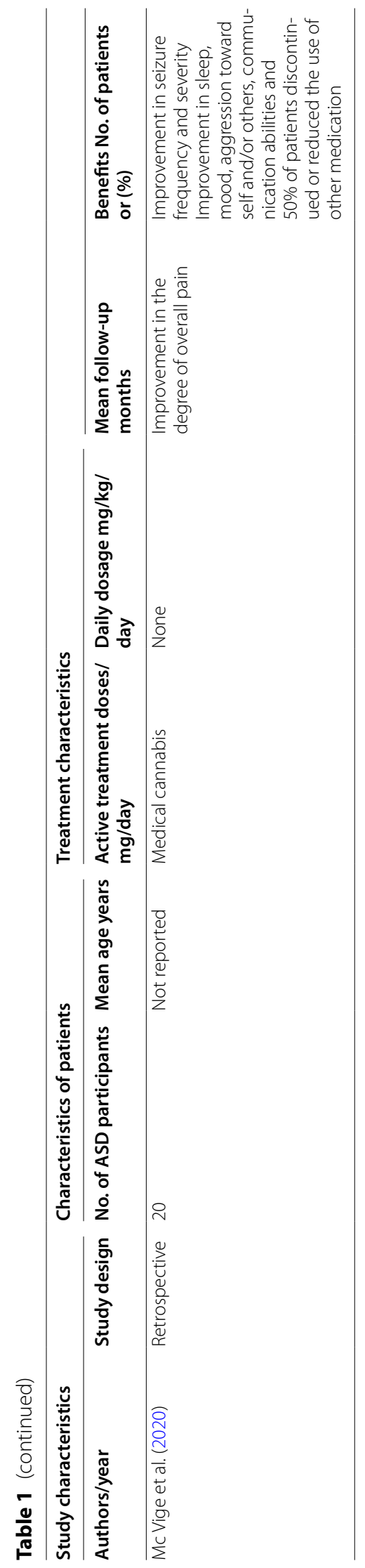




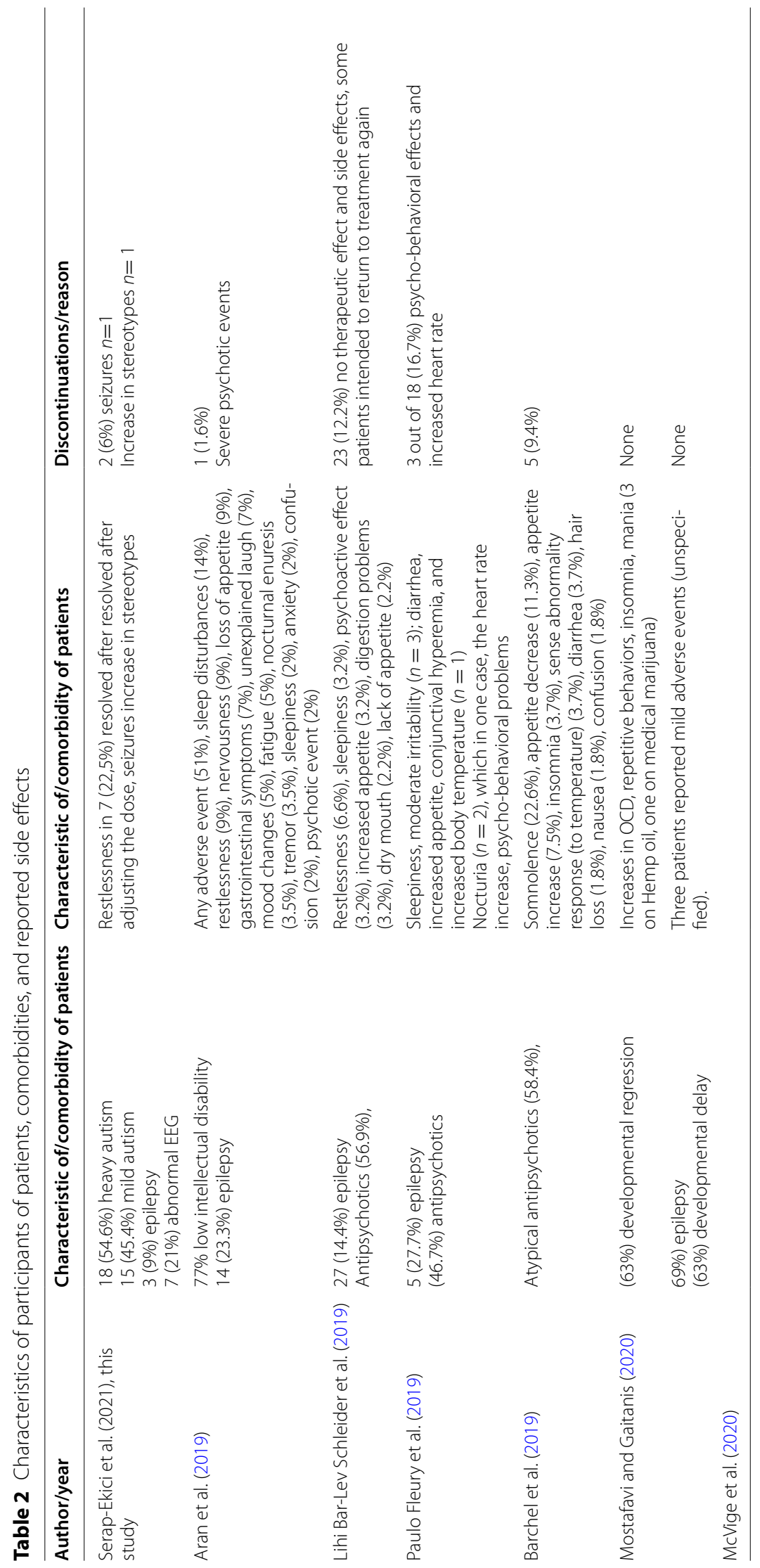




\section{Abbreviations}

ASD: Autism spectrum disorder; ECS: Endocannabinoid system; CBD: Cannabidiol; THC: Tetrahydrocannabinol; FDA: Food and Drug Administration; eCBs: Endocannabinoids; GPR: G protein-coupled receptor; CB: Cannabinoid receptor; TRPV1: Transient receptor potential cation channel subfamily $\vee$ member 1; FAAH: Fatty acid amide hydrolase; PPAR- $\gamma$ : Peroxisome proliferator-activated receptor-gamma; GPR55: G Protein-coupled receptor 55; ROS: Reactive oxygen species; OEA: Oleoylethanolamide; MC4R: Melanocortin 4 receptor; NAc: Nucleus accumbens; ACTH: Adrenocorticotropic hormone; CaGl: Caregiver Global Impression of Change; EEG: Electroenceography.

\section{Acknowledgements}

The authors are grateful to the editorial committee for their kindness in submitting this review article and our experience to the journal.

\section{Authors' contributions}

Bilge S. and EKICI B. contributed equally to the acquisition, analysis, and interpretation of the data and the drafting and revision of this review. The authors have read and approved the submitted version.

\section{Funding}

No funding has been received

\section{Availability of data and materials}

The datasets used and analyzed in this review article are available from the corresponding author upon reasonable request.

\section{Declarations}

\section{Ethics approval and consent to participate}

Not applicable. Written informed consent was obtained from the guardians.

\section{Consent for publication}

Not applicable.

\section{Competing interests}

The authors declare that they have no competing interests.

\section{Author details}

${ }^{1}$ Department of Pediatric Neurology, Çukurova Medical School, 01790 Balcalı, Turkey. ${ }^{2}$ Department of Pediatric Neurology, Pediatric Neurology Clinics, Istanbul, Turkey.

Received: 11 March 2021 Accepted: 8 November 2021

Published online: 16 December 2021

\section{References}

Agrawal A, Lynskey MT. Candidate genes for cannabis use disorders: findings, challenges and directions. Addiction. 2009;104:518-32.

Aran A, Cassuto H, Lubotzky A, Wattad N, Hazan E. Cannabidiol-rich cannabis in children with autism spectrum disorder and severe behavioral problems-a retrospective feasibility study. J Autism Dev Disord. 2019:49:1284-8.

American Psychiatric Association (APA). DSM-V Diagnostic and Statistical Manual of Mental Disorders. 5th Edition. Washington, DC: American Psychiatric Association; 2013.

Barchel D, Stolar O, De-Haan T, Ziv-Baran T, Saban N, Fuchs D, et al. Oral cannabidiol use in children with autism spectrum disorder to treat related symptoms and co-morbidities. Front Pharmacol. 2019;9:1521.

Bartova A, Birmingham MK. Effect of delta 9-tetrahydrocannabinol on mitochondrial NADH-oxidase activity. J Biol Chem. 1976;251:5002-6.

Bloomfield MAP, Ashok AH, Volkow ND, Howes OD. Effect of delta9-tetrahydrocannabinol on the synthesis of dopamine and norepinephrine in Mouse brain synaptosomes. Pharmacol Exp Ther. 1982;221:97-103.

Bridgemanan MB, Abazia DT. Medicinal cannabis: history, pharmacology, and implications for the acute care setting. P T. 2017;423:180-8.
Castillo PE, Younts TJ, Chávez AE, Hashimotodani Y. Endocannabinoid signaling and synaptic function. Neuron. 2012;76:70-81. https://doi.org/10.1016/j. neuron.2012.09.020.

Davies C, Bhattacharyya S. Cannabidiol as a potential treatment for psychosis. Ther Adv Psychopharmacol. 2019;9:2045125319881916.

Dos Santos RG, Flávia LO, Rocio MS, Antonio WZ, Jaime E, Cecilio H, et al. Modulation of the endocannabinoid and oxytocinergic systems as a potential treatment approach for social anxiety disorder. CNS Drugs. 2019.

Fusar-Poli L, Vito C, Tinacci S, Concas I, Petralia A, Signorelli MS, et al. Cannabinoids for people with ASD: a systematic review of published and ongoing studies. Brain Sci. 2020;9:572-6.

Leas EC, Hendrickson M, Nobles LA, Davey RT, Smith M, Dredze M, et al. Selfreported cannabidiol (CBD) use for conditions with proven therapies. JAMA Netw Open. 2020;3(10):e2020977.

Lihi Bar-Lev Schleider, Mechoulam R, Saban N, Meiri G, Novack V. Real-life experience of medical cannabis treatment in autism: analysis of safety and efficacy. Sci Rep. 2019;9:1-3.

Maenner MJ, Shaw K, Baio J, Washington A, Patrick M. Prevalence of autism spectrum disorder among children aged 8 years. Autism Dev Disabil Monit Netw. 2020:69:1-12.

Mc Partlan JM, Guy GW, Di Marzo V. Care and feeding of the endocannabinoid system: a systematic review of potential clinical interventions that upregulate the endocannabinoid system. PLoS One. 2014;9:3.

McVige J, Headd V, Alwahaidy M, Lis D, Kaur D, Albert B, et al. Medical cannabis in the treatment of patients with autism spectrum disorder. 2020:94:1648.

Mostafavi M, Gaitanis J. Autism spectrum disorder and medical cannabis: review \& clinical experience. Semin Pediatr Neurol. 2020;35:100833.

Paulo Fleury T, Caixeta FV, Ramires da Silva LC, Brasil-Neto JP, Malcher-Lopes R. Effects of CBD-enriched cannabis sativa extract on autism spectrum disorder symptoms: an observational study of 18 participants undergoing compassionate use. Front Neurol. 2019;10:1145.

Premolia M, Francesca A, Boninia SA, Maccarinellia G, Gianoncellia A, Pinaa SD, et al. Cannabidiol: recent advances and new insights for neuropsychiatric disorders treatment. Life Sci. 2019;224:120-7.

Pretzsch CM, Freyberg J, Voinescu B, Lythgoe D, Horder J, Andreina MMR, et al. Effects of cannabidiol on brain excitation and inhibition systems; a randomized placebo-controlled single-dose trial during magnetic resonance spectroscopy in adults with and without autism spectrum disorder. Neuropsychopharmacology. 2019a;44:1398-405.

Pretzsch CM, Voinescu B, Mendez MA, Wichers R, Ajram L, Ivin G, et al. The effect of cannabidiol (CBD) on low-frequency activity and functional connectivity in the brain of adults with and without autism spectrum disorder (ASD). J Psychopharmacol. 2019b;33:1141.

Rodríguez De Fonseca F, Fernández-Ruiz J, Murphy L, Cebeira M, Steger R, Bartke AW, et al. Acute effects of delta-9-tetrahydrocannabinol on dopaminergic activity in several rat brain areas. Pharmacol Biochem Behav. 1992:42:269-75.

Russo EB, Burnett A, Hall B, Parker K. Agonistic properties of cannabidiol at 5 HT1a receptors. Neurochem Res. 2005;30:1037-43.

Ryan D, Drysdale AJ, Lafourcade C, Pertwee RG, Platt B. Cannabidiol targets mitochondria to regulate intracellular Ca2+ levels. J Neurosci. 2009;29:2053-63

Weia D, Lee Y, Coxa CD, Karsten CA, Peñagarikanob O. Endocannabinoid signaling mediates oxytocin-driven social reward. PNAS. 2015;45:14084-9.

Zamberletti E, Gabaglio M, Parolaro D. The endocannabinoid system and autism spectrum disorders: insights from animal models. J Mol Sci. 2017;18:1916.

\section{Publisher's Note}

Springer Nature remains neutral with regard to jurisdictional claims in published maps and institutional affiliations. 